Christine Lorut

Aurélie Lefebvre

Benjamin Planquette

Laurent Quinquis

Hervé Clavier

Nicola Santelmo

Halim Abou Hanna

François Bellenot

Jean-François Regnard

Marc Riquet

Pierre Magdeleinat

Guy Meyer

Nicolas Roche

Marie-Pierre Revel

Gérard Huchon

Joel Coste

Antoine Rabbat

\section{Erratum to: Early postoperative prophylactic noninvasive ventilation after major lung resection in COPD patients: a randomized controlled trial}

Published online: 25 January 2014

(C) Springer-Verlag Berlin Heidelberg and ESICM 2014

The online version of the original article can be found under doi:10.1007/s00134-013-3150-2.

C. Lorut $(\varangle) \cdot$ A. Lefebvre · N. Roche - G. Huchon · A. Rabbat Service de pneumologie et USIR, Department of Respiratory and Intensive Care Medicine, Cochin-Broca-Hôtel-Dieu Hospital Group, Site Cochin, AP-HP, University Paris5, René Descartes,

27 rue du Faubourg Saint Jacques, 75679 Paris cedex 14, France e-mail: christine.lorut@cch.aphp.fr

Tel.: +33-1-58412089

Fax: +33-1-58412088

J.-F. Regnard

Department of Thoracic Surgery, Cochin-Broca-Hôtel-Dieu Hospital Group, AP-HP, University Paris5, René Descartes, Paris, France

L. Quinquis · J. Coste

Department of Biostatistics and Epidemiology, Hôtel-Dieu Hospital, AP-HP, University Paris5,

René Descartes, Paris, France

B. Planquette - G. Meyer

Department of Respiratory and Intensive Care Medicine, European Georges Pompidou Hospital, AP-HP, University Paris5, René Descartes, Paris, France

\section{Riquet}

Department of Thoracic Surgery, European Georges Pompidou Hospital, AP-HP, University Paris5, René Descartes, Paris, France

\section{H. Clavier}

Department of intensive care Medicine, Institut Montsouris

Hospital, Paris, France
P. Magdeleinat

Department of Thoracic Surgery, Institut Montsouris Hospital, Paris, France

\section{N. Santelmo}

Department of Thoracic Surgery, University Hospital, Strasbourg, France

H. A. Hanna

Department of Thoracic Surgery, University Hospital,

Dijon, France

F. Bellenot

Department of Thoracic Surgery, University Hospital,

Pontoise, France

M.-P. Revel

Department of Radiology, Cochin-Broca-Hôtel-Dieu Hospital Group, AP-HP, University Paris5, René Descartes, Paris, France

\section{Erratum to: Intensive Care Med DOI 10.1007/s00134-013-3150-2}

The co-author Marie-Pierre Revel was omitted from the manuscript supplied for publication; this error is corrected here. 